\title{
EQUAÇÕES LINEARES E NÃO LINEARES PARA PREVER O COMPORTAMENTO DE PROPRIEDADES FÍSICO-QUÍMICAS DE COMBUSTÍVEL DE AVIAÇÃO MISTURADO COM BIOQUEROSENE DROP-IN ALTERNATIVO
}

Bruno K. V. Iha ${ }^{\mathrm{a}, *}$, Leila Ribeiro dos Santos ${ }^{\mathrm{b}}$, Lenilson Afonso dos Santos ${ }^{\mathrm{a}}$, Maria Esther Sbampato ${ }^{\mathrm{b}}$ e José A. F. F. Rocco

aDepartamento de Química, Instituto Tecnológico de Aeronáutica, 12228-900 São José dos Campos - SP, Brasil

${ }^{\mathrm{b}}$ Departamento de Propulsão, Instituto Tecnológico de Aeronáutica, 12228-900 São José dos Campos - SP, Brasil

Recebido em 04/05/2018; aceito em 24/09/2018; publicado na web em 31/10/2018

\begin{abstract}
LINEAR AND NON-LINEAR EQUATIONS TO PREDICT THE BEHAVIOR OF PHYSICO-CHEMICAL PROPERTIES OF AVIATION FUEL MIXED WITH ALTERNATIVE BIOQUEROSENE DROP-IN. Density, viscosity and calorific values are important physical properties to affect the utilization of biofuel. In this work, mixtures of farnesane and a "jet fuel", QAv-1, were used to study the variation of density and kinematic viscosity as a function of the volumetric fraction of farnesane and temperature. Experimental measurements were carried out for nineteen farnesane blends and pure farnesane and QAv-1, at four temperatures in the range of 293 - 323 K. Variation of high heating (HHV) and low heating (LHV) values were also determined for different blends of farnesane/ QAv-1. Several empirical correlations were used to predict the density, the kinematic viscosity, HHV and LHV values. By using some of these empirical correlations, the estimated values of those three properties studied are in excellent agreement with the experimental data, with low absolute average prediction error.
\end{abstract}

Keywords: farnesane; jet-fuel drop in; density; viscosity; heating values.

\section{INTRODUÇÃO}

A indústria da aviação mundial representa $2 \%$ das emissões globais de dióxido de carbono $\left(\mathrm{CO}_{2}\right)$ (Associação Internacional do Transporte Aéreo - IATA, 2011) e, se não forem tomadas quaisquer medidas, as emissões triplicarão até 2050. A indústria da aviação tem grande interesse em participar de um esforço global para mitigar as emissões de gases de efeito estufa e, portanto, está comprometida em reduzir as emissões de $\mathrm{CO}_{2}$. O objetivo atual é uma redução de $50 \%$ das emissões de $\mathrm{CO}_{2}$ em relação aos níveis de 2005. . $^{1-4}$

A atividade de pesquisa científica e tecnológica no segmento de aviação é forçada pelas demandas de consumo de querosene em razão do crescimento do segmento de aviação em todo o mundo e, particularmente, no Brasil. ${ }^{5}$ As possíveis fontes de substituição, fósseis ou renováveis, envolvem produtos cujas especificações devem atender aos mais elevados padrões de qualidade para combustíveis de aviação. O governo brasileiro tem se mobilizado para contribuir com um ambiente propício à pesquisa de biocombustíveis que sejam compatíveis com o querosene de aviação mineral, aproveitando o grande potencial que o país apresenta em biomassa e sua longa tradição em combustíveis renováveis. ${ }^{6}$

Os denominados biocombustíveis drop-in são combustíveis que podem ser misturados com querosene de aviação convencional até uma proporção específica, permitindo que possa ser empregada a mesma infraestrutura de abastecimento já existente, não havendo necessidade de adaptação das aeronaves e suas turbinas. ${ }^{7,8}$

O bioquerosene (farnesano) utilizado neste trabalho é uma tecnologia da empresa Amyris ${ }^{\circledR}$ que mudou geneticamente a levedura utilizada na fermentação da cana de açúcar, de forma que o produto seja um hidrocarboneto denominado farneseno. $\mathrm{O}$ farneseno $\left(\mathrm{C}_{15} \mathrm{H}_{24}\right)$ pode ser transformado em produtos para a indústria química (p. ex., óleos industriais e automotivos e lubrificantes) ou em combustíveis para transporte. Para ser utilizado como biocombustível, o farneseno é hidrogenado, dando origem ao farnesano $\left(\mathrm{C}_{15} \mathrm{H}_{32}\right)$, que apresenta

*e-mail: bkviha@gmail.com características semelhantes ao querosene de aviação (QAv-1).

O conhecimento da variação de propriedades físicas, como densidade, viscosidade e poder calorífico de misturas biocombustível/ combustível, é importante uma vez que impactam diretamente no desempenho de motores. Na literatura, há diversos modelos e equações que permitem prever as variações de densidade, viscosidade e poder calorífico em função da fração de biocombustível na mistura. ${ }^{9}$ Geralmente, com um número relativamente pequeno de dados experimentais, pode-se fazer o ajuste das curvas e encontrar os parâmetros das equações-modelo. Com estas equações, pode-se calcular o valor das propriedades citadas em diferentes condições experimentais, tais como temperatura e/ou fração de biocombustível na mistura.

O presente trabalho tem por objetivo a determinação da variação de importantes propriedades físicas de misturas de QAv-1 com farnesano. As propriedades estudadas foram a densidade, a viscosidade e o poder calorífico superior e inferior. Foram avaliadas diversas misturas, entre $95 \%$ e $5 \%$ de farnesano, em temperaturas entre 293 K e $323 \mathrm{~K}$, e estabelecidos parâmetros de equações-modelo que tornam possível o cálculo das propriedades estudadas em diferentes condições experimentais de concentração e temperatura.

\section{PARTE EXPERIMENTAL}

A Amyris Biotechnologies Inc. sediada na Califórnia, USA, com uma planta-demo no Brasil (Campinas, SP) produz o farneseno num processo de fermentação dos açúcares presentes no caldo da cana-de-açúcar. Esta fermentação envolve várias reações enzimáticas e modificações genéticas da levedura Saccharomyces cerevisiae. A etapa seguinte promove a hidrogenação do farneseno e sua transformação em farnesano, $\mathrm{C}_{15} \mathrm{H}_{32}$, um terpeno também denominado 2, 6, 10-trimetilduodecano, cuja fórmula estrutural é apresentada no Esquema 1. A etapa final é a destilação para a separação do farnesano, com a finalidade de obter um produto considerado puro, com cerca de $95 \%$ de farnesano. O produto é um líquido de cor amarelada que pode conter enxofre em teores menores que $3 \mathrm{mg} \mathrm{kg}^{-1}$ mas ausência de compostos aromáticos. ${ }^{10}$ 
<smiles>CCC(C)CCCC(C)CCCC(C)C</smiles>

Esquema 1. Estrutura molecular do farnesano

O querosene de aviação QAv-1 é uma mistura de hidrocarbonetos líquidos obtidos a partir do fracionamento do petróleo. É uma mistura complexa formada por hidrocarbonetos alifáticos, naftênicos e aromáticos, com número de átomos de carbono entre C8 e C16, com pontos de ebulição entre 150 e $290^{\circ} \mathrm{C}$. É uma fração do petróleo intermediária à gasolina e o diesel. Considerando-se todos os componentes do QAv-1, suas proporções em massa e a respectiva análise estrutural, pode-se estabelecer uma fórmula molecular média, ${ }^{11} \mathrm{C}_{11} \mathrm{H}_{21}$, com massa molar igual a $153,3 \mathrm{~g} \mathrm{~mol}^{-1}$.

Para a preparação das misturas utilizou-se farnesano doado pela empresa Amyris ${ }^{\circledR}$ e QAv-1 comercial, pertencendo cada um deles a um único lote. As misturas foram preparadas em balões volumétricos de 1,00 L, em quantidade suficiente para realizar todos os ensaios, cobrindo a faixa de $5 \%$ a $95 \%$ em volume de farnesano, em um total de 19 misturas. A densidade e a viscosidade foram medidas a $293 \mathrm{~K}$; $303 \mathrm{~K} ; 313 \mathrm{~K}$ e $323 \mathrm{~K}$. Preliminarmente, foram feitas medições em triplicata para cada técnica e condição experimental.

Para determinação da densidade das amostras, utilizou-se um equipamento certificado - densímetro digital automático de bancada Rudolph Research Analytical, modelo DDM 2911- Série 3.5, com módulo Peltier para controle de temperatura. A amostra é injetada através de uma seringa em um capilar de volume constante. Durante o procedimento de medição (completamente automatizado), aguarda-se o equilíbrio térmico da amostra na temperatura ajustada e o dado de densidade é fornecido após alguns minutos, considerando uma pressão de 1,1013 bar. O equipamento é rotineiramente calibrado e apresenta um desvio padrão de $\pm 0,00001 \mathrm{~g} \mathrm{~cm}^{-3}$, garantindo um grau de confiança de $95 \%$.

A medição da viscosidade das amostras foi realizada utilizando-se um viscosímetro modelo LAUDA Proline PV15, com módulo Peltier para o controle da temperatura. A determinação de viscosidades cinemáticas envolve o uso de viscosímetros capilares padronizados. A escolha do capilar adequado foi feita com base nos valores de viscosidade das misturas estimados com base nos valores das viscosidades médias do farnesano e do QAv-1. A constante do capilar de vidro utilizado é igual a $0,01 \mathrm{~mm}^{2} \mathrm{~s}^{-2}$. A lavagem dos capilares foi feita com hexano seguida do enxague com a própria amostra. As medições de viscosidade foram feitas em triplicata.

Para a determinação do poder calorífico, foi utilizado o calorímetro da marca IKA, modelo C 1. Este equipamento é um calorímetro de combustão e mede o poder calorífico superior (PCS) de substâncias líquidas e sólidas não explosivas. As amostras são queimadas em um recipiente metálico fechado, com oxigênio sob pressão, a uma temperatura inicial padrão de $22,8{ }^{\circ} \mathrm{C}$. A quantidade de calor liberada, medida em um sistema previamente calibrado com pastilhas de ácido benzóico, permite o cálculo do PCS. Utilizou-se cerca de $0,5 \mathrm{~g}$ de amostra em cada determinação, pesando-se diretamente no cadinho utilizado para a queima, por meio de uma balança analítica da Quimis, modelo Q500B210C, com precisão de $\pm 0,0001$ g. O poder calorífico da amostra é calculado considerando-se o peso da amostra combustível, a capacidade calorífica do calorímetro e a variação da temperatura da água. As determinações foram feitas em triplicata.

\section{RESULTADOS E DISCUSSÃO}

Para verificar a possibilidade de se prever com exatidão as propriedades físicas das misturas em diferentes concentrações e temperaturas, contando com um número pequeno de determinações experimentais ou apenas com as propriedades dos componentes puros, alguns modelos matemáticos foram estudados e encontram-se descritos a seguir.

\section{Densidade das misturas de farnesano com QAv-1}

A Tabela 1S (Material Suplementar) apresenta os valores experimentais de densidade $\left(\mathrm{g} \mathrm{cm}^{-3}\right)$ para farnesano e QAv-1 puros e misturas farnesano/QAv-1 em diferentes proporções, em diferentes temperaturas.

A densidade diminui em função do aumento de temperatura, o que pode ser atribuído ao ganho de energia térmica das moléculas da mistura. A densidade está relacionada com a força de atração entre as moléculas do líquido; com o ganho de energia térmica as moléculas conseguem se afastar mais umas das outras e, consequentemente, a densidade diminui. A análise dos dados experimentais mostra, também, que a densidade diminui com o aumento da fração de farnesano. Esta variação é justificada considerando que a densidade do farnesano é menor que a densidade do querosene.

\section{Variação da densidade com a fração volumétrica de farnesano}

O primeiro modelo estudado considera que a densidade de misturas de dois ou mais componentes em uma dada temperatura pode ser calculada considerando as densidades individuais dos componentes da mistura e as respectivas frações, dadas pela razão volume do componente/volume total ou pela porcentagem. Para uma mistura binária, a densidade a uma certa temperatura é dada por: ${ }^{9}$

$$
\rho_{\mathrm{m}}=\rho_{1} \cdot X_{1}+\rho_{2} \cdot X_{2}
$$

em que $\rho_{\mathrm{m}}$ é a densidade da mistura, $X_{1}$ e $X_{2}$ são as frações volumétricas dos componentes 1 e 2 , e $\rho_{1}$ e $\rho_{2}$, as respectivas densidades.

Utilizando os valores de densidade do farnesano e do querosene e a equação (1), os valores das densidades das misturas em diferentes proporções de farnesano foram calculados. Os erros relativos percentuais foram obtidos usando a equação (2) e, com os resultados obtidos para cada temperatura, os respectivos desvios médios absolutos (dma) foram calculados conforme a equação (3):

$$
\begin{gathered}
E_{\%}=\frac{x_{i}-y_{i}}{x_{i}} .100 \\
\operatorname{dma}(\%)=\frac{100}{n} \sum_{1}^{n} \frac{\left|x_{i}-y_{i}\right|}{x_{i}}
\end{gathered}
$$

em que $x_{i}$ e $y_{i}$ representam, respectivamente, o valor experimental e o valor calculado com a equação (1).

Os valores de dma (\%) foram calculados para cada conjunto de 19 frações de farnesano $(n=19)$ e os seguintes resultados foram obtidos para cada temperatura: $\operatorname{dma}(293 \mathrm{~K})=0,02 \%$; $\operatorname{dma}(303 \mathrm{~K})=0,02 \%$; $\mathrm{dma}(313 \mathrm{~K})=0,03 \%$ e dma $(323 \mathrm{~K})=0,03 \%$. Desta forma, verifica-se que os valores de densidade calculados com a equação (1) apresentam excelente concordância com os valores experimentais.

Considerando que a densidade varia linearmente em função da fração de farnesano, os dados experimentais foram ajustados a uma equação de primeiro grau, $\mathrm{y}=\mathrm{a} \mathrm{x}+\mathrm{b}$, em que $\mathrm{b}$ representa a densidade do QAv-1 puro. Para obtenção dos parâmetros $a$ e $b$, além do coeficiente de correlação, aplicou-se o método dos mínimos quadrados (regressão linear). Os resultados estão apresentados na Tabela 1. Neste caso, os valores de dma (\%) foram calculados para o conjunto de dados de densidade em cada temperatura, resultando todos iguais a $0,02 \%$, tornando evidente a excelente concordância entre os valores experimentais e os calculados. A Tabela 1 apresenta, 
Tabela 1. Valores dos parâmetros de correlação a, b e $\mathrm{r}^{2}$ e dma (\%) obtidos usando o modelo da equação de reta $(\mathrm{y}=\mathrm{a} \mathrm{x}+\mathrm{b})$ para os dados de densidade das misturas de farnesano e QAv-1. Valores experimentais e calculados para o farnesano e o querosene puros

\begin{tabular}{ccccccc}
\hline$T(\mathrm{~K})$ & $\mathrm{a}$ & $\mathrm{b}$ & $\mathrm{r}^{2}$ & $\mathrm{dma}(\%)$ & $\begin{array}{c}\text { Farnesano } \\
\left.(\mathrm{g} \mathrm{cm})^{-3}\right) \\
\exp / \mathrm{calc}^{*}\end{array}$ & $\begin{array}{c}\mathrm{QAv}-1 \\
(\mathrm{~g} \mathrm{~cm} \\
\left.\mathrm{exp} / \mathrm{calc}^{-3}\right)\end{array}$ \\
\hline 293 & $-0,0347$ & 0,80311 & 0,9996 & 0,02 & $0,7687 / 0,7684$ & $0,8030 / 0,8031$ \\
303 & $-0,0340$ & 0,79560 & 1,000 & 0,02 & $0,7616 / 0,7616$ & $0,7956 / 0,7956$ \\
313 & $-0,0340$ & 0,78819 & 0,9995 & 0,02 & $0,7545 / 0,7542$ & $0,7882 / 0,7882$ \\
323 & $-0,0336$ & 0,78072 & 0,9994 & 0,02 & $0,7475 / 0,7471$ & $0,7807 / 0,7807$ \\
\hline
\end{tabular}

*valores calculados, com a respectiva equação de reta, para farnesano e querosene puros.

ainda, a excelente concordância entre os valores de densidade experimentais e calculados para os componentes puros.

A comparação entre os valores de densidade das misturas farnesano/QAv-1 calculados com a equação (1) e a equação de primeiro grau mostra que para ambas a correlação com os dados experimentais é excelente, como verificado pelos valores de dma iguais a $0,02 \%$ ou $0,03 \%$.

\section{Variação da densidade com a temperatura e a fração de farnesano nas misturas com $Q A v-1$}

Um modelo simples para calcular a densidade de misturas binárias de biodiesel/diesel como uma função da temperatura e da fração do biodiesel foi estabelecida por Ramirez-Verduzco et al. ${ }^{12}$ Esse modelo é representado pela seguinte equação:

$$
\rho_{\mathrm{m}}=\mathrm{a} \cdot \mathrm{X}+\mathrm{b} \cdot \mathrm{T}+\mathrm{c}
$$

onde a, b e c são os parâmetros ajustáveis da equação, $X$ é a fração volumétrica do biocombustível na mistura e $\mathrm{T}$ é a temperatura em Kelvin (K). Aplicando-se este modelo para o sistema farnesano/ QAv-1, os parâmetros da equação (4) foram estimados utilizando os 76 dados experimentais de densidade (Tabela $1 \mathrm{~S}$ ) relativos às misturas (não foram incluídos os dados para o farnesano e o QAv-1 puros). Os parâmetros encontrados são apresentados na equação 4(a):

$$
\rho_{\mathrm{m}}=-3,4164 \cdot 10^{-4} \cdot X_{\mathrm{fms}}-7,2747 \cdot 10^{-4} \cdot \mathrm{T}+1,0161
$$

Usando a equação 4(a), os valores de densidade foram calculados para as 76 misturas de farnesano/QAv-1 e comparados com os dados experimentais. A correlação entre os dados calculados e os experimentais é excelente, sendo comprovada com o cálculo do coeficiente de correlação $r^{2}$ que resultou igual a 0,9996. Os erros relativos percentuais foram calculados e, a partir deles, obteve-se o valor do desvio médio absoluto (dma) igual a $0,002 \%$, o que confirma o excelente ajuste e estabelece a possibilidade de se calcular a densidade de misturas com proporções de farnesano e temperaturas diferentes das utilizadas experimentalmente.

A Figura 1 mostra a variação dos erros relativos percentuais dos valores de densidade calculados. Os erros percentuais encontram-se numa faixa entre $-0,07 \%$ e $+0,06 \%$. Pode-se verificar a presença de erros sistemáticos positivos nos valores extremos de densidade. Estes erros foram observados para valores de densidade maiores que $0,80 \mathrm{~g} \mathrm{~cm}^{-3}$ (que correspondem a frações volumétricas de farnesano abaixo de $15 \%$ a $293 \mathrm{~K}$ ), e valores menores que $0,76 \mathrm{~g} \mathrm{~cm}^{-3}$ (que correspondem a frações maiores que $70 \%$ de farnesano a $323 \mathrm{~K}$ ). Para a faixa intermediária, que compreende a maior parte dos pontos experimentais, a distribuição de erros relativos é equilibrada.

\section{Viscosidade cinemática de misturas de farnesano com QAv-1}

A viscosidade cinemática ou coeficiente de viscosidade é a medida da resistência oferecida por uma substância em fluir e tem

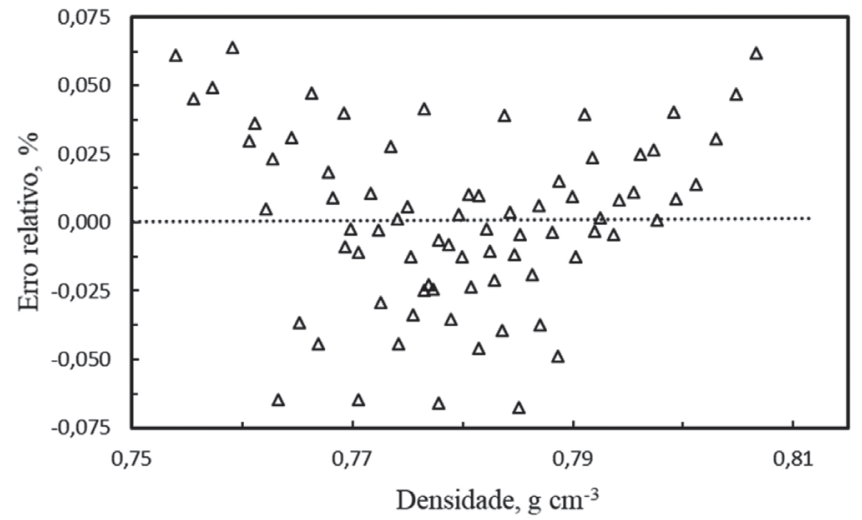

Figura 1. Erros relativos percentuais de densidade de misturas de farnesano/ QAv-1 calculada em função da densidade experimental

como origem a atração intermolecular entre as moléculas do fluído. Geralmente, a viscosidade cinemática de um biocombustível é mais alta quando comparada à do diesel, por exemplo. ${ }^{13}$ As viscosidades mais altas afetam as características de aerossol do combustível, ocasionando uma atomização pobre e formação de gotas maiores, o que pode causar deposição de carbono nos filtros de combustível e nas válvulas de injeção. Uma vantagem de se utilizar misturas biocombustível/combustível pode ser a otimização da viscosidade para melhorar o desempenho de um motor. Assim, torna-se importante avaliar a variação da viscosidade para diferentes composições das misturas biocombustível/combustível e diferentes temperaturas. Com esse propósito, alguns valores de viscosidade devem ser determinados experimentalmente e a partir deles, utilizando alguns modelos matemáticos, ${ }^{9}$ pode-se fazer a previsão dos valores de viscosidade cinemática para outras temperaturas e composições das misturas.

A Tabela $2 \mathrm{~S}$ apresenta os valores experimentais de viscosidade cinemática $\left(\mathrm{mm}^{2} \mathrm{~s}^{-1}\right)$ e os respectivos desvios padrão para farnesano e QAv-1 puros e misturas farnesano/QAv-1 em diferentes proporções e temperaturas. A viscosidade cinemática aumenta com o aumento da fração de farnesano e diminuiu com o aumento da temperatura. $\mathrm{O}$ aumento da viscosidade em função da fração de farnesano pode ser explicado pelo fato de a viscosidade do farnesano ser maior que a do QAv-1.

\section{Variação da viscosidade cinemática com a fração volumétrica de farnesano}

Os modelos de equações ${ }^{13-15}$ que relacionam a viscosidade cinemática com a fração volumétrica de farnesano na mistura com querosene, utilizados no presente trabalho, encontram-se na Tabela 2. Os parâmetros de cada equação foram encontrados fazendo-se o ajuste dos dados experimentais pelo método de regressão por mínimos quadrados.

A Tabela 2 apresenta os parâmetros a, b e c das equações (5) e (6) e os valores de desvio médio absoluto (dma), além da amplitude dos erros percentuais dos valores de viscosidade cinemática calculados com aquelas equações. Pode-se observar que os coeficientes 
Tabela 2. Modelos utilizados para o estudo da variação de viscosidade cinemática (v) com a fração volumétrica $(\mathrm{X})$ de cada componente nas misturas com QAv-1. Parâmetros das equações (5) e (6) e os respectivos coeficientes de correlação. Valores de dma e amplitude dos erros percentuais para o cálculo da viscosidade usando as equações (5) a (7).

\begin{tabular}{|c|c|c|c|c|c|c|c|c|}
\hline \multirow{2}{*}{$T(\mathrm{~K})$} & \multicolumn{4}{|c|}{ Parâmetros das equações } & \multirow{2}{*}{$\operatorname{dma}(\%)$} & \multirow{2}{*}{\multicolumn{2}{|c|}{ Amplitude de Erro (\%) }} & \multirow{2}{*}{ Referência } \\
\hline & $\mathrm{a}$ & $\mathrm{b}$ & $\mathrm{c}$ & $r^{2}$ & & & & \\
\hline \multicolumn{5}{|c|}{ Equação (5): $v=a+b \cdot X^{c}$} & & & & 13 \\
\hline 293 & 1,8306 & 0,001381 & 1,5564 & 0,9973 & 0,7 & 1,6 & 0,007 & \\
\hline 303 & 1,5630 & 0,001560 & 1,4684 & 0,9981 & 0,6 & 1,5 & 0,0 & \\
\hline 313 & 1,3491 & 0,001492 & 1,4231 & 0,9969 & 0,7 & 1,4 & 0,03 & \\
\hline 323 & 1,1892 & 0,001405 & 1,3847 & 0,9978 & 0,5 & 1,5 & 0,02 & \\
\hline \multicolumn{5}{|c|}{ Equação (6): $v=a+b \cdot X+c \cdot X^{2}$} & & & & 14 \\
\hline 293 & 1,8000 & 0,007214 & $1,1144 \times 10^{-4}$ & 0,9987 & 0,5 & 1,3 & 0,01 & \\
\hline 303 & 1,5403 & 0,006504 & $7,3250 \times 10^{-5}$ & 0,9992 & 0,4 & 1,0 & 0,04 & \\
\hline 313 & 1,3320 & 0,005485 & $5,2531 \times 10^{-5}$ & 0,9983 & 0,5 & 1,3 & 0,02 & \\
\hline 323 & 1,1760 & 0,004644 & $3,8289 \times 10^{-5}$ & 0,9988 & 0,4 & 1,2 & 0,02 & \\
\hline \multicolumn{5}{|c|}{ Equação (7): $\ln v=X_{1} \cdot \ln v_{1}+X_{2} \cdot \ln v_{2}$} & & & & 15 \\
\hline 293 & - & - & - & - & 3,2 & 5,1 & 0,3 & \\
\hline 303 & - & - & - & - & 2,1 & 3,8 & 0,06 & \\
\hline 313 & - & - & - & - & 2,4 & 4,0 & 0,8 & \\
\hline 323 & - & - & - & - & 2,0 & 3,6 & 0,2 & \\
\hline
\end{tabular}

de correlação encontrados para a aplicação das equações (5) e (6) evidenciam uma ótima concordância entre os valores calculados e os experimentais. Por outro lado, pode-se observar que no caso da aplicação da equação (7) não há uma concordância satisfatória entre os valores de viscosidade calculados e os experimentais, relativos a cada fração de farnesano, em todas as temperaturas utilizadas. No caso da equação (7), os erros encontrados são maiores variando de 0,2 a $5,1 \%$.

A Figura 2 apresenta a variação dos valores experimentais (símbolos) de viscosidade cinemática em função da fração volumétrica porcentual de farnesano para diferentes temperaturas. Nesta figura, também é apresentada a variação dos valores de viscosidade calculados com as equações (5), (6) e (7). É possível verificar a concordância menor entre os dados experimentais (símbolos) e os calculados usando a equação (7) e uma concordância excelente com o uso das equações (5) e (6).

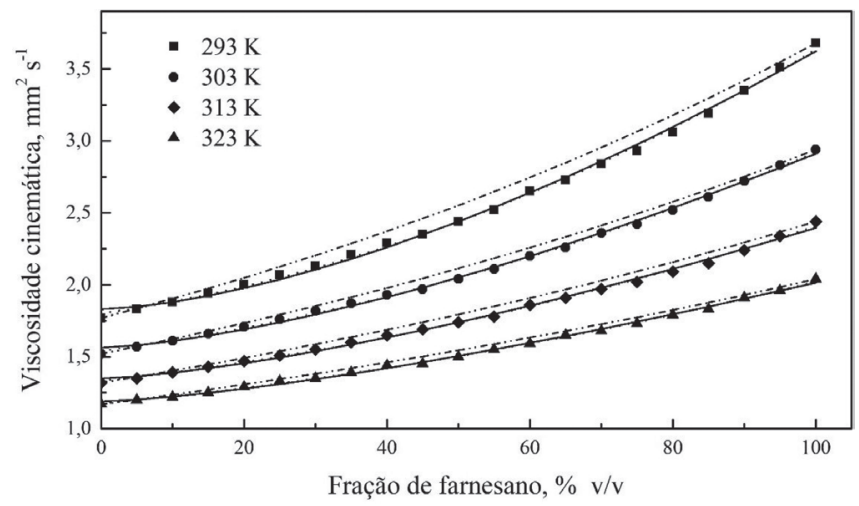

Figura 2. Valores experimentais (símbolos) e calculados (linhas) de viscosidade cinemática em função da fração volumétrica de farnesano — Eq. (5) ---- Eq. (6) -..-Eq. (7)

\section{Variação da viscosidade cinemática das misturas farnesano/QAv-1} com a temperatura

O aumento da temperatura leva a uma diminuição nas forças de atração coesiva e aumenta a energia cinética das moléculas, provocando a diminuição da viscosidade. Os modelos de equações, ${ }^{13,16-18}$ que relacionam a viscosidade cinemática com a temperatura, utilizados no presente trabalho, encontram-se na Tabela 3. Os parâmetros de cada equação foram calculados fazendo-se o ajuste dos dados experimentais por um método de regressão por mínimos quadrados. Além dos modelos encontrados na literatura, os dados experimentais foram ajustados por regressão polinomial de grau 2 .

A Tabela 3 apresenta os parâmetros a, b e c das equações (8) a (12), os coeficientes de correlação entre os valores de viscosidade cinemática experimentais e calculados e a amplitude dos erros percentuais para cada equação e temperatura. Estão apresentados apenas os valores relativos às frações de farnesano variando de $20 \mathrm{em} 20$ unidades, entretanto, os cálculos foram efetuados para todos os 76 dados experimentais.

De acordo com os resultados apresentados na Tabela 3, excelentes coeficientes de correlação foram encontrados para as equações (8), (10) e (12). A mesma observação se aplica também quando se analisam os valores dos erros percentuais. No caso das equações exponenciais (9) e (11), foram encontrados erros relativos percentuais significativamente maiores e, além disso, observa-se uma oscilação em função da temperatura, com erros negativos para $293 \mathrm{~K}$ e $323 \mathrm{~K}$ e positivos para $303 \mathrm{~K}$ e $313 \mathrm{~K}$.

De acordo com os valores apresentados, considerando que com a equação (12) obtiveram-se os menores erros relativos e que esses erros não foram sistematicamente positivos ou negativos para cada temperatura (Tabela 3), pode-se concluir que a equação polinomial de grau 2, equação (12), é a que representa com maior exatidão a correlação entre a viscosidade cinemática das misturas e a temperatura.

\section{Variação da viscosidade cinemática em função da fração volumétrica de farnesano e da temperatura}

Para relacionar a variação da viscosidade cinemática de uma mistura biocombustível / combustível com a temperatura e a fração do biocombustível simultaneamente, foram aplicados seis modelos matemáticos, ${ }^{12,19-21}$ apresentados na Tabela 4.

Considerando as equações apresentadas, (13) a (18), os seis modelos foram aplicados para determinar os parâmetros que relacionam a viscosidade cinemática, das misturas de farnesano/querosene, com 
Tabela 3. Modelos utilizados para o estudo da variação da viscosidade cinemática (v) com a temperatura (T). Parâmetros das equações (8) a (12), coeficientes de correlação e os erros relativos percentuais dos valores de viscosidade calculados, em função da temperatura.

\begin{tabular}{|c|c|c|c|c|c|c|c|c|}
\hline \multirow{2}{*}{$\mathrm{X}_{\mathrm{Fns}}, \%$} & \multicolumn{4}{|c|}{ Parâmetros dos ajustes } & \multicolumn{4}{|c|}{ Erros rel. (\%) } \\
\hline & $\mathrm{a}$ & $\mathrm{b}$ & $\mathrm{c}$ & $r^{2}$ & $293 \mathrm{~K}$ & $303 \mathrm{~K}$ & $313 \mathrm{~K}$ & $323 \mathrm{~K}$ \\
\hline \multicolumn{9}{|c|}{ Equação (8): $v=a+b \cdot T^{c} 13$} \\
\hline 20 & 4,830 & $-1,356$ & 0,2456 & 0,9997 & $-0,05$ & 0,305 & $-0,41$ & 0,23 \\
\hline 40 & 8,970 & $-4,509$ & 0,1312 & 0,9999 & $-0,03$ & 0,222 & $-0,29$ & 0,18 \\
\hline 60 & 9,793 & $-4,542$ & 0,1511 & 1,0000 & $-0,04$ & 0,006 & $-0,14$ & $-0,04$ \\
\hline 80 & 18,90 & $-12,29$ & 0,08474 & 0,9996 & $-0,08$ & 0,447 & $-0,68$ & 0,36 \\
\hline \multicolumn{9}{|c|}{ Equação (9): v = a.exp(b/T) ${ }^{16}$} \\
\hline 20 & 1,041 & 13,381 & - & 0,9532 & $-1,59$ & 4,933 & 1,08 & $-5,43$ \\
\hline 40 & 1,145 & 14,175 & - & 0,9586 & $-1,54$ & 4,875 & 1,13 & $-5,54$ \\
\hline 60 & 1,245 & 15,427 & - & 0,9560 & $-1,64$ & 5,329 & 1,53 & $-6,64$ \\
\hline 80 & 1,378 & 16,290 & - & 0,9572 & $-1,71$ & 5,864 & 0,90 & $-6,65$ \\
\hline \multicolumn{9}{|c|}{ Equação (10): $v=\exp a+b / T+c / T^{2} 17$} \\
\hline 20 & $-0,4243$ & 41,983 & $-392,80$ & 0,9996 & 0,03 & $-0,221$ & 0,55 & $-0,36$ \\
\hline 40 & $-0,3289$ & 42,726 & $-391,58$ & 0,9996 & 0,01 & $-0,268$ & 0,63 & $-0,43$ \\
\hline 60 & $-0,3051$ & 47,627 & $-440,83$ & 0,9990 & 0,04 & $-0,421$ & 1,05 & $-0,74$ \\
\hline 80 & $-0,2368$ & 50,452 & $-467,01$ & 0,9998 & 0,02 & $-0,176$ & 0,45 & $-0,32$ \\
\hline \multicolumn{9}{|c|}{ Equação (11): $v=a \cdot T^{b} 18$} \\
\hline 20 & 8,1422 & $-0,4655$ & - & 0,9906 & $-0,94$ & 2,243 & 0,54 & $-2,16$ \\
\hline 40 & 10,115 & $-0,4930$ & - & 0,9929 & $-0,86$ & 2,008 & 0,53 & $-2,10$ \\
\hline 60 & 13,409 & $-0,5381$ & - & 0,9916 & $-0,94$ & 2,244 & 0,96 & $-2,75$ \\
\hline 80 & 16,990 & $-0,5689$ & - & 0,9919 & $-1,00$ & 2,621 & 0,31 & $-2,52$ \\
\hline \multicolumn{9}{|c|}{ Equação (12): $v=a+b \cdot T+c \cdot T^{2}$} \\
\hline 20 & 2,7495 & $-0,04295$ & $2,750.10^{-4}$ & 1,0000 & $-0,02$ & 0,088 & $-0,10$ & 0,04 \\
\hline 40 & 3,2305 & $-0,05455$ & $3,750.10^{-4}$ & 1,0000 & 0,02 & $-0,078$ & 0,09 & $-0,03$ \\
\hline 60 & 3,8020 & $-0,06670$ & $4,500.10^{-4}$ & 0,9999 & 0,08 & $-0,273$ & 0,32 & $-0,13$ \\
\hline 80 & 4,5090 & $-0,08440$ & $6,000.10^{-4}$ & 1,0000 & $-0,03$ & 0,119 & $-0,14$ & 0,06 \\
\hline
\end{tabular}

a fração e a temperatura. Os valores dos 76 dados experimentais de viscosidade cinemática, obtidos em função da fração volumétrica de farnesano (X) e da temperatura (T) para as misturas farnesano/ QAv-1, foram utilizados.

A Tabela 4 reúne os valores encontrados (a, b, c e d) para as equações (13) a (18). São apresentados também os respectivos coeficientes de correlação $\left(r^{2}\right)$, os valores do desvio médio absoluto (dma) e a amplitude dos erros percentuais. Pode-se observar que os coeficientes de correlação são muito bons para os seis modelos empregados, $r^{2} \geq 0,994$.

Utilizando os seis modelos de correlação apresentados na Tabela 4 , os valores de viscosidade e os respectivos erros relativos foram calculados para as 76 misturas farnesano/QAv-1. Pode-se observar que os valores mais baixos de dma foram obtidos com as equações (14) e (16). Por outro lado, os dados apresentados na mesma tabela permitem estabelecer que os modelos relativos às equações (13) e (17) não são adequados. Apesar de os valores de dma serem perfeitamente aceitáveis, a faixa de valores de erro relativo é significativamente alta. Tem-se o intervalo de $+7,7 \%$ a $-2,3 \%$ para equação (13) e $+5,7 \%$ a $-2,1 \%$ para a equação (17)

A Figura 3 apresenta a variação dos erros relativos em função da viscosidade cinemática experimental. Conforme se observa nas Figuras 3(a) e 3(e) relativas ao uso das equações (13) e (17), são evidentes os erros sistemáticos nos valores calculados da viscosidade cinemática. Considerando a faixa total de valores experimentais de viscosidade $\left(v=1,17 \mathrm{~mm}^{2} \mathrm{~s}^{-1}\right.$ a $\left.3,68 \mathrm{~mm}^{2} \mathrm{~s}^{-1}\right)$, tem-se que para valores mais baixos $\left(v \leq 1,5 \mathrm{~mm}^{2} \mathrm{~s}^{-1}\right)$ e mais altos $\left(v \geq 2,9 \mathrm{~mm}^{2} \mathrm{~s}^{-1}\right)$ de viscosidade, os erros são sistematicamente positivos. Na faixa intermediária, a maioria dos erros é negativa.

As Figuras 3(c) e 3(f)) apresentam uma distribuição ligeiramente melhor que as Figuras (a) e (e), mas também apresentam faixas com erros sistemáticos. No caso das Figuras 3(b) e 3(d), a distribuição dos erros relativos é equilibrada considerando-se os erros positivos e negativos. Dessa forma, os modelos que utilizam as equações (14) e (16) são os que melhor representam a variação da viscosidade cinemática em função da temperatura e da fração de farnesano nas misturas com QAv-1.

A Figura 4 apresenta a correlação entre os valores de viscosidade cinemática experimentais e os calculados com a equação (14). O coeficiente de correlação $\left(\mathrm{r}^{2}\right)$ encontrado é igual a 0,998 e confirma a excelente concordância obtida entre os valores calculados e os experimentais. Cabe ressaltar que a mesma conclusão foi confirmada com o uso da equação (16).

\section{Poder calorífico de misturas farnesano com QAv-1}

O poder calorífico representa a quantidade de calor liberada durante a combustão completa de um combustível e, consequentemente, 
Tabela 4. Modelos utilizados para o estudo da variação da viscosidade cinemática (v) com a temperatura (T) e a fração volumétrica (X) de farnesano. Parâmetros das equações (13) a (18) e os respectivos coeficientes de correlação, $\mathrm{r}^{2}$. Valores de dma e amplitude dos erros percentuais para o cálculo da viscosidade cinemática, em função da temperatura e da fração de farnesano nas misturas com QAv-1

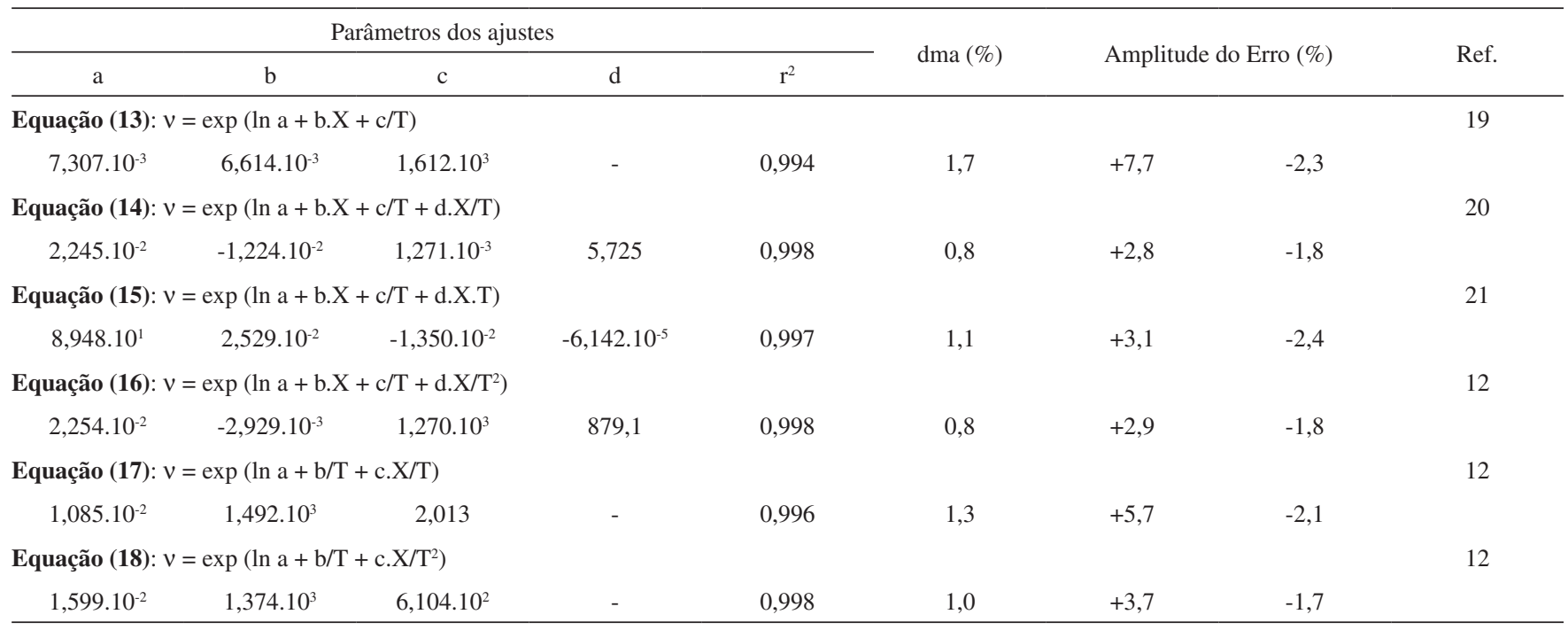
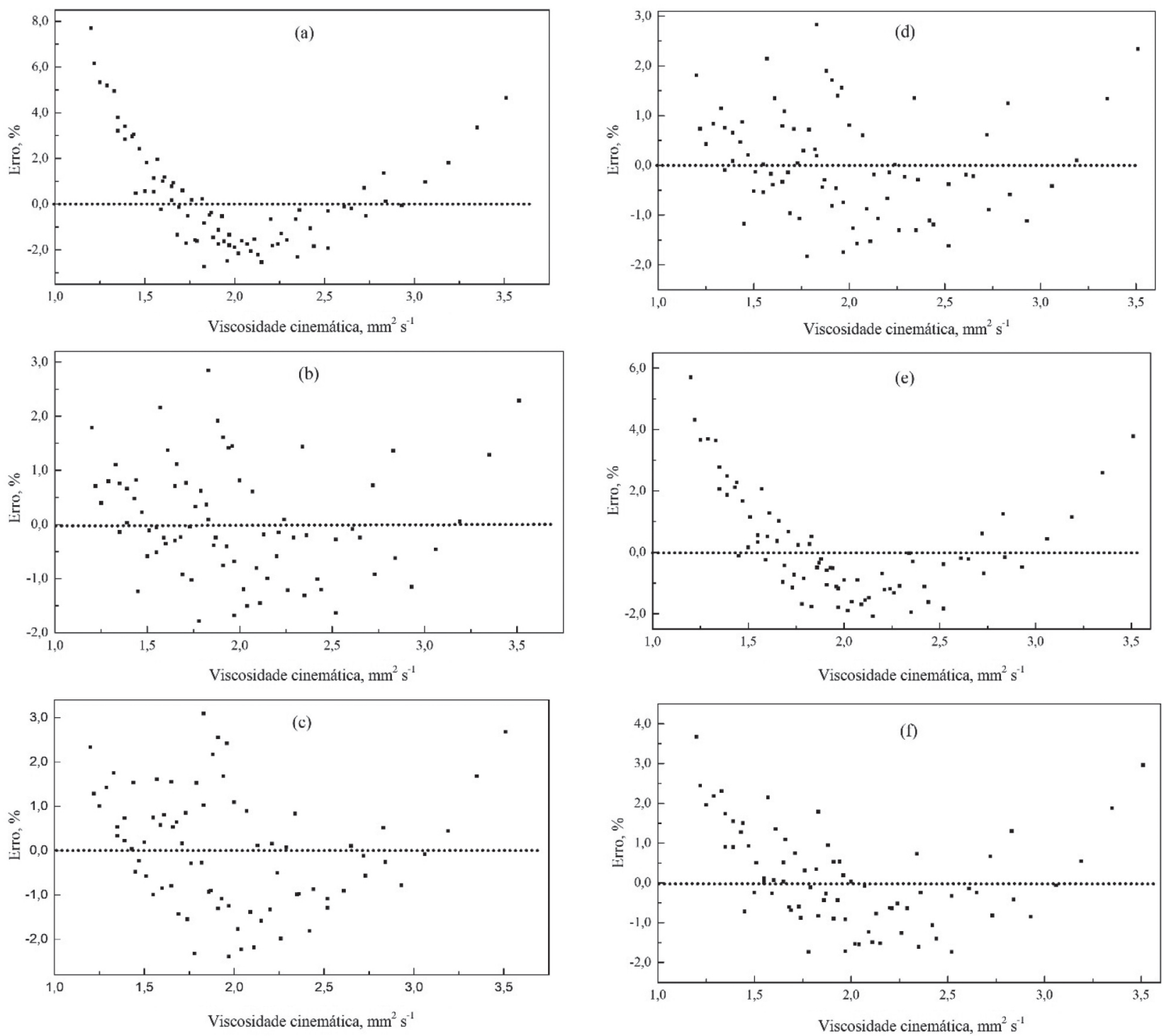

Figura 3. Erros relativos dos valores de viscosidade cinemática calculados para as misturas de farnesano com QAv-1, em função da viscosidade experimental; (a) Eq.(13); (b) Eq.(14); (c) Eq.(15); (d) Eq.(16); (e) Eq.(17); (f) Eq.(18) 


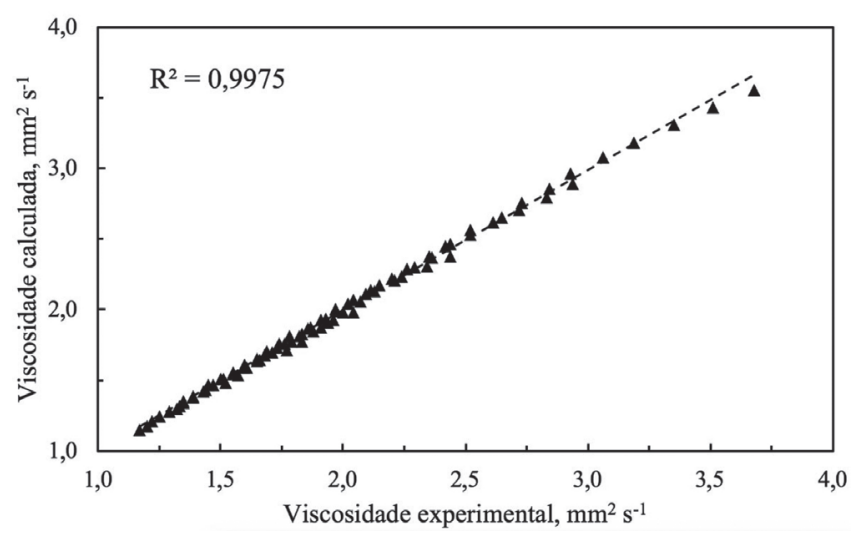

Figura 4. Correlação entre os valores de viscosidade cinemática calculados com a equação (14) e os valores experimentais

adquire um valor que varia de acordo com a composição química do combustível. Há dois tipos de poder calorífico, classificados como superior (PCS) e inferior (PCI). O primeiro corresponde à quantidade de calor produzida por grama de combustível quando este sofre combustão completa, em excesso de ar, e os gases de descarga são resfriados de modo que o vapor de água formado seja condensado (o calor contido no vapor de água é recuperado). O segundo equivale à quantidade de calor que um grama de combustível pode produzir quando sofre combustão completa, com excesso de ar, e a água é produzida e mantida no estado de vapor. ${ }^{22}$

As reações de combustão completa do farnesano, Fns, e do querosene de aviação, QAv-1, são representadas pelas seguintes equações químicas:

farnesano: $\mathrm{C}_{15} \mathrm{H}_{32}+23 \mathrm{O}_{2} \rightarrow 15 \mathrm{CO}_{2}+16 \mathrm{H}_{2} \mathrm{O}$

QAV-1: $\mathrm{C}_{11} \mathrm{H}_{21}+65 / 4 \mathrm{O}_{2} \rightarrow 11 \mathrm{CO}_{2}+21 / 2 \mathrm{H}_{2} \mathrm{O}$

Variação do poder calorífico superior com a fração volumétrica de farnesano

A Tabela 5 apresenta a média dos valores experimentais de poder calorífico superior (PCS) determinados em função da fração volumétrica porcentual de farnesano $\left(\mathrm{X}_{\mathrm{v}, \mathrm{Fns}}\right)$. Os valores das frações em massa do farnesano na mistura são também apresentados nesta tabela, $\mathrm{X}_{\mathrm{m}, \mathrm{Fns}}=\frac{X_{\mathrm{v}, \mathrm{Fns}} \cdot \rho_{\mathrm{Fns}}}{X_{\mathrm{v}, \mathrm{Fns}} \cdot \rho_{\mathrm{Fns}}+\left(1-X_{\mathrm{v}, \mathrm{Fns}}\right) \cdot \rho_{\mathrm{QAV}-1}}$. Pode-se observar que os valores de PCS aumentam em função do aumento da fração de farnesano, variando de 45793 (100\% de QAV-1) a $46946 \mathrm{~J} \mathrm{~g}^{-1}$ (100\% de farnesano).

Os valores experimentais de PCS variam linearmente em função da fração volumétrica $(X)$ de farnesano na mistura. Desta forma, os dados foram ajustados a uma equação de reta e os seguintes parâmetros foram obtidos:

$$
\mathrm{PCS}=1263,8 \cdot \mathrm{X}+45734 ; \mathrm{r}^{2}=0,98
$$

Para efeito de comparação, os valores de PCS foram calculados através da equação (21) e encontram-se na Tabela 5. Os erros relativos percentuais foram obtidos para cada resultado e a partir de seus valores absolutos calculou-se o valor do dma, que resultou em $0,08 \%$. O intervalo dos erros relativos é - $0,22 \%$ a $+0,15 \%$.

O poder calorífico superior para cada mistura pode também ser calculado usando a seguinte equação: ${ }^{22}$

$$
\mathrm{PCS}=\mathrm{X}_{\mathrm{m}, \mathrm{Fns}} \cdot \mathrm{PCS}_{\mathrm{Fns}}+\left(1-\mathrm{X}_{\mathrm{m}, \mathrm{Fns}}\right) \cdot \mathrm{PCS}_{\mathrm{QAV}-1}
$$

em que os subíndices Fns e QAv-1 representam o farnesano e o QAv-1 na mistura e $X_{m}$, como já definido, representa a fração do farnesano em massa. As propriedades do farnesano e do QAv-1 necessárias ao cálculo dos valores de PCS são os valores de PCS para componentes puros (46946 $\mathrm{J} \mathrm{g}^{-1}$ para o farnesano e $45793 \mathrm{~J} \mathrm{~g}^{-1}$ para o QAv-1) e para o cálculo das frações em massa as densidades a $293 \mathrm{~K}$ são $0,7687 \mathrm{~g} \mathrm{~cm}^{-3}$ para o farnesano e $0,8030 \mathrm{~g} \mathrm{~cm}^{-3}$ para o QAv-1.

A Tabela 5 reúne os valores experimentais e os calculados usando a equação (22) e os erros relativos percentuais. Observa-se uma concordância excelente entre os valores calculados e experimentais evidenciada também pelo valor do desvio médio absoluto (dma) resultante igual a $0,10 \%$. O intervalo dos erros relativos é - $0,2 \%$ e $+0,3 \%$. Os resultados obtidos são, também, semelhantes aos obtidos pelo ajuste dos dados experimentais a uma equação de reta, conforme consta na Tabela 5.

A comparação entre todos os valores de PCS calculados com as equações (21) e (22) mostra que em todos os casos a correlação com os dados experimentais é muito boa, como demonstrado pelos valores de dma iguais, respectivamente, a $0,08 \%$; e $0,10 \%$.

\section{Variação do poder calorífico inferior com a fração volumétrica de} farnesano

Como definido anteriormente, o poder calorífico inferior (PCI) equivale à quantidade de calor que um grama de combustível pode produzir quando entra em combustão, com excesso de ar, e a água produzida é mantida no estado de vapor, ou seja, é o calor útil da combustão. O PCI de uma substância é calculado descontando-se do valor do PCS obtido experimentalmente, a contribuição do calor de vaporização da água.

De acordo com as equações (19) e (20), observa-se que para cada mol de farnesano e QAv-1 queimados são produzidos, respectivamente, 16 e 21/2 mol de água. Sabendo-se que as massas moleculares dos componentes das misturas são $212,4 \mathrm{~g} \mathrm{~mol}^{-1}$ para o farnesano e $153,3 \mathrm{~g} \mathrm{~mol}^{-1}$ para o QAv-1, serão obtidos $1,356 \mathrm{~g}$ de água na queima de $1 \mathrm{~g}$ de farnesano e $1,234 \mathrm{~g}$ de água na queima de $1 \mathrm{~g}$ de QAv-1. O calor de vaporização da água, $\mathrm{C}_{\mathrm{v}(\text { agua) }}$, é igual a $540 \mathrm{cal} \mathrm{g}^{-1}$ ou $2259 \mathrm{~J} \mathrm{~g}^{-1} \mathrm{e}$, deste modo, o PCI experimental das misturas $\left(\mathrm{J} \mathrm{g}^{-1}\right)$ pode ser calculado através de:

$\mathrm{PCI}=\mathrm{PCS}-\mathrm{X}_{\mathrm{m}, \mathrm{Fns}} \cdot 1,356 \cdot \mathrm{C}_{\mathrm{v}(\text { água })}-\left(1-\mathrm{X}_{\mathrm{m}, \mathrm{Fns}}\right) \cdot 1,234 \cdot \mathrm{C}_{\mathrm{v}(\text { água })}$

A Tabela 6 apresenta os valores de PCI experimentais calculados. Da mesma forma que os valores de PCS experimentais, os valores de PCI apresentam uma correlação linear com as frações de farnesano. Assim, os dados foram ajustados a uma equação de reta e os seguintes parâmetros foram obtidos:

$$
\text { PCI }=967,38 \cdot X+42969 ; r^{2}=0,97
$$

Os valores de PCI das misturas de farnesano com QAv-1 foram obtidos, também, a partir dos valores de PCI dos componentes puros utilizando-se a equação (25):

$$
\mathrm{PCI}=\mathrm{X}_{\mathrm{m}, \mathrm{Fns}} \cdot \mathrm{PCI}_{\mathrm{Fns}}+\left(1-\mathrm{X}_{\mathrm{m}, \mathrm{Fns}}\right) \cdot \mathrm{PCI}_{\mathrm{QAV}-1}
$$

em que $\mathrm{PCI}_{\mathrm{Fns}}$ é $43883 \mathrm{~J} \mathrm{~g}^{-1}$ e $\mathrm{PCI}_{\mathrm{QAV}-1}, 43005 \mathrm{~J} \mathrm{~g}^{-1}$ (calculados através das medições experimentais, Tabela 6).

A Tabela 6 apresenta os valores de PCI calculados com as equações (24) e (25) e os respectivos erros relativos percentuais. Observa-se uma concordância excelente entre os valores calculados com as duas equações e os experimentais. $\mathrm{O}$ valor do desvio médio absoluto (dma) calculado para os conjuntos de dados obtidos com ambas as equações resultou igual a $0,09 \%$. 
Tabela 5. Média dos valores experimentais e o desvio padrão de poder calorífico superior e valores calculados usando as equações (21) e (22)

\begin{tabular}{|c|c|c|c|c|c|c|}
\hline \multicolumn{2}{|c|}{ Farnesano } & \multirow{2}{*}{$\begin{array}{c}\text { PCS experimental } \\
\left(\mathrm{J} \mathrm{g}^{-1}\right)\end{array}$} & \multicolumn{2}{|c|}{$\begin{array}{l}\text { PCS calculado } \\
\text { Equação (21) }\end{array}$} & \multicolumn{2}{|c|}{$\begin{array}{l}\text { PCS calculado } \\
\text { Equação (22) }\end{array}$} \\
\hline$\% \mathrm{v} / \mathrm{v}$ & $\mathrm{X}_{\mathrm{m}, \mathrm{Fns}}$ & & $\left(\mathrm{J} \mathrm{g}^{-1}\right)$ & Erro $(\%)$ & $\left(\mathrm{J} \mathrm{g}^{-1}\right)$ & Erro $(\%)$ \\
\hline 0 & 0 & $45793 \pm 92$ & 45734 & 0,130 & & \\
\hline 5 & 0,04796 & $45795 \pm 33$ & 45797 & $-0,005$ & 45848 & -0.116 \\
\hline 10 & 0,09613 & $45868 \pm 45$ & 45860 & 0,017 & 45904 & -0.078 \\
\hline 15 & 0,14451 & $45897 \pm 12$ & 45924 & $-0,059$ & 45960 & -0.138 \\
\hline 20 & 0,19309 & $45981 \pm 46$ & 45987 & $-0,013$ & 46016 & -0.076 \\
\hline 25 & 0,24189 & $46111 \pm 54$ & 46050 & 0,131 & 46072 & 0.084 \\
\hline 30 & 0,29090 & $46131 \pm 23$ & 46113 & 0,039 & 46128 & 0.006 \\
\hline 35 & 0,34011 & $46178 \pm 16$ & 46176 & 0,004 & 46185 & -0.015 \\
\hline 40 & 0,38955 & $46293 \pm 28$ & 46240 & 0,115 & 46242 & 0.109 \\
\hline 45 & 0,43920 & $46311 \pm 44$ & 46303 & 0,017 & 46299 & 0.024 \\
\hline 50 & 0,48907 & $46324 \pm 55$ & 46366 & $-0,091$ & 46357 & -0.072 \\
\hline 55 & 0,53915 & $46330 \pm 35$ & 46429 & $-0,215$ & 46415 & -0.183 \\
\hline 60 & 0,58946 & $46505 \pm 9$ & 46492 & 0,027 & 46473 & 0.070 \\
\hline 65 & 0,63998 & $46571 \pm 11$ & 46555 & 0,033 & 46531 & 0.085 \\
\hline 70 & 0,69073 & $46689 \pm 38$ & 46619 & 0,150 & 46589 & 0.213 \\
\hline 75 & 0,74171 & $46638 \pm 48$ & 46682 & $-0,095$ & 46648 & -0.023 \\
\hline 80 & 0,79291 & $46721 \pm 8$ & 46745 & $-0,051$ & 46707 & 0.030 \\
\hline 85 & 0,84434 & $46735 \pm 18$ & 46808 & $-0,157$ & 46767 & -0.068 \\
\hline 90 & 0,89599 & $46960 \pm 84$ & 46871 & 0,189 & 46826 & 0.285 \\
\hline 95 & 0,94788 & $46993 \pm 54$ & 46935 & 0,124 & 46886 & 0.227 \\
\hline 100 & 1,0000 & $46946 \pm 28$ & 46998 & $-0,110$ & & \\
\hline
\end{tabular}

Tabela 6. Dados experimentais de poder calorífico inferior em função da fração de farnesano e valores calculados usando as equações (24) e (25)

\begin{tabular}{|c|c|c|c|c|c|c|}
\hline \multicolumn{2}{|c|}{ Farnesano } & \multirow{2}{*}{$\begin{array}{l}\text { PCI experimental } \\
\qquad\left(\mathrm{J} \mathrm{g}^{-1}\right)\end{array}$} & \multicolumn{2}{|c|}{$\begin{array}{l}\text { PCI calculado } \\
\text { Equação (24) }\end{array}$} & \multicolumn{2}{|c|}{$\begin{array}{l}\text { PCI calculado } \\
\text { Equação (25) }\end{array}$} \\
\hline$\% \mathrm{v} / \mathrm{v}$ & $X_{\mathrm{m}, \mathrm{Fns}}$ & & $\left(\mathrm{J} \mathrm{g}^{-1}\right)$ & Erro $(\%)$ & $\left(\mathrm{J} \mathrm{g}^{-1}\right)$ & Erro $(\%)$ \\
\hline 0 & 0 & 43005 & 42969 & 0,085 & 43005 & 0,001 \\
\hline 5 & 0,04796 & 42994 & 43015 & $-0,049$ & 43047 & $-0,123$ \\
\hline 10 & 0,09613 & 43054 & 43062 & $-0,019$ & 43089 & $-0,082$ \\
\hline 15 & 0,14451 & 43069 & 43109 & $-0,092$ & 43132 & $-0,146$ \\
\hline 20 & 0,19309 & 43140 & 43156 & $-0,037$ & 43175 & $-0,080$ \\
\hline 25 & 0,24189 & 43256 & 43203 & 0,123 & 43217 & 0,090 \\
\hline 30 & 0,29090 & 43263 & 43250 & 0,030 & 43260 & 0,007 \\
\hline 35 & 0,34011 & 43297 & 43298 & $-0,002$ & 43304 & $-0,015$ \\
\hline 40 & 0,38955 & 43398 & 43346 & 0,119 & 43347 & 0,117 \\
\hline 45 & 0,43920 & 43402 & 43394 & 0,018 & 43391 & 0,026 \\
\hline 50 & 0,48907 & 43401 & 43442 & $-0,094$ & 43434 & $-0,076$ \\
\hline 55 & 0,53915 & 43393 & 43491 & $-0,224$ & 43478 & $-0,196$ \\
\hline 60 & 0,58946 & 43555 & 43539 & 0,036 & 43523 & 0,074 \\
\hline 65 & 0,63998 & 43607 & 43588 & 0,043 & 43567 & 0,091 \\
\hline 70 & 0,69073 & 43711 & 43637 & 0,168 & 43611 & 0,227 \\
\hline 75 & 0,74171 & 43646 & 43687 & $-0,094$ & 43656 & $-0,024$ \\
\hline 80 & 0,79291 & 43715 & 43736 & $-0,048$ & 43701 & 0,032 \\
\hline 85 & 0,84434 & 43714 & 43786 & $-0,163$ & 43746 & $-0,073$ \\
\hline 90 & 0,89599 & 43925 & 43836 & 0,204 & 43792 & 0,305 \\
\hline 95 & 0,94788 & 43944 & 43886 & 0,132 & 43837 & 0,243 \\
\hline 100 & 1,0000 & 43883 & 43936 & $-0,122$ & 43883 & 0,000 \\
\hline
\end{tabular}




\section{CONCLUSÕES}

A densidade das misturas, conforme o esperado, diminui com o aumento da fração de farnesano pois sua densidade é menor que a densidade do QAv-1. Diminui também em função do aumento da temperatura. A relação entre a densidade e a fração de farnesano nas misturas com QAv-1 mostrou excelentes resultados tanto com a equação de reta quanto com a equação da mistura. Considera-se vantajosa a equação da mistura, pois não é necessário fazer ajustes para encontrar os parâmetros; esta equação depende unicamente da consideração das frações volumétricas dos componentes da mistura e de suas densidades. Com relação à variação da densidade em função da temperatura e da fração de farnesano, os valores de densidade calculados apresentaram uma excelente concordância com os dados experimentais. Os erros relativos resultaram bastante baixos ficando entre $-0,07 \%$ e $+0,06 \%$.

A viscosidade das misturas farnesano/QAv-1 aumenta com o aumento da fração volumétrica de farnesano e diminui com o aumento da temperatura. A relação entre a viscosidade e a fração volumétrica de farnesano nas misturas mostrou excelentes resultados com a equação polinomial de grau 2, com desvios médios absolutos da ordem de 0,4\% (para $303 \mathrm{~K}$ e $323 \mathrm{~K}$ ) e $0,5 \%$ (para $293 \mathrm{~K}$ e $313 \mathrm{~K}$ ).

A previsão da variação da viscosidade em relação à fração de farnesano e à temperatura foi testada com aplicação de seis equações. Duas equações exponenciais levaram a resultados similares e satisfatórios. Nos dois casos, o valor de dma foi $0,8 \%$. No caso destas duas equações observou-se também uma distribuição equilibrada dos erros relativos positivos e negativos.

O poder calorífico é um parâmetro importante na seleção de um combustível. Este valor determina a energia química do combustível a qual específica a eficiência da combustão. O poder calorífico depende de várias características e, entre elas, o tamanho da cadeia carbônica. No caso das misturas de farnesano/QAv-1, considerando as fórmulas moleculares do farnesano, $\mathrm{C}_{15} \mathrm{H}_{32}$, e do QAv-1, $\mathrm{C}_{11} \mathrm{H}_{21}$, o aumento dos valores de PCS e PCI pode ser associado ao fato de o aumento da fração de farnesano representar um número maior de átomos de carbono.

\section{MATERIAL SUPLEMENTAR}

A Tabela $1 \mathrm{~S}$ apresenta os valores experimentais de densidade ( $\mathrm{g}$ $\mathrm{cm}^{-3}$ ) para farnesano e QAV-1 puros e misturas farnesano/QAv-1 em diferentes proporções, em diferentes temperaturas.

A Tabela $2 \mathrm{~S}$ apresenta os valores experimentais de viscosidade cinemática $\left(\mathrm{mm}^{2} \mathrm{~s}^{-1}\right)$ para farnesano e QAv-1 puros e misturas farnesano/QAv-1 em diferentes proporções e temperaturas. São apresentados também os valores de desvio padrão dos valores de viscosidade cinemática.

O material suplementar associado a este artigo pode ser consultado na versão online em: http://quimicanova.sbq.org.br/, com livre acesso.

\section{AGRADECIMENTOS}

Os autores agradecem à Amyris ${ }^{\circledR}$ pelo fornecimento das amostras de farnesano, à CAPES e ao CNPq.

\section{REFERÊNCIAS}

1. Bailis, R. E.; Baka, J. E.; Environ. Sci. Technol. 2010, 44, 8684.

2. Hileman, J. I.; Blanco, E. R.; Bonnefoy, P. A.; Carter, N. A.; Progress in Aerospace Sciences 2013, 63, 84.

3. http://www.iata.org/pressroom/facts_figures/Documents/vision-2050.pdf, acessada em junho de 2017.

4. Kousoulidou, M.; Lonza, L.; Transportation Research Part D 2016, 46, 166.

5. Cremonez, P. A.; Feroldi, M.; Araújo, A. V.; Borges, M. N.; Meier, T. W.; Feiden, A.; Teleken, J. G.; Renewable Sustainable Energy Rev. 2015, 43, 1063.

6. https://www.cgee.org.br/serie-documentos-tecnicos, acessada em agosto de 2017.

7. Pires, A. P. P.; Han, Y.; Kramlich, J.; Garcia-Perez, M.; BioResources 2018, 13, 2632.

8. Karatzos, S.; McMillan, J. D.; Saddler, J. N.; IEA Bioenergy Task 39 The Potential and Challenges of Drop-in Biofuels, 2014, 203 p.

9. Gaonkar, N.; Vaidya, R. G. Em Renewable Energy Systems; Kale, S. A., ed.; Nova Science Publishers, Inc.: New York, 2017, cap. 9.

10. Millo, F.; Bensaid, S.; Fino, D.; Marcano, S. J. C.; Vlachos, T.; Debnath, B. K.; Fuel 2014, 138, 134.

11. Edwards, T.; Maurice, L. Q.; J. Propul. Power 2001, 17, 461.

12. Ramírez-Verduzco, L. F.; García-Flores, B. E.; Rodríguez-Rodríguez, J. E.; Jaramillo-Jacob, A. R.; Fuel 2011, 90, 1751.

13. Gülüm, M.; Bilgin, A.; Fuel Process. Technol. 2016, 149, 121.

14. Alptekin, E.; Canakci, M.; Renewable Energy 2008, 33, 2623.

15. Grunberg, L.; Nissan, A.; Nature 1949, 164, 799.

16. Jouyban, A.; Khoubnasabjafari, M.; Gharamaleki, Z. V.; Fekari, Z.; Acree Jr., W. E.; Chem. Pharm. Bull. 2005, 53, 519.

17. Benjumea, P.; Agudelo, J.; Agudelo, A.; Fuel 2008, 87, 2069.

18. Fasina, O. O.; Hallman, H.; Craig-Schmidt, M.; Clements, C.; J. Am. Oil Chem. Soc. 2006, 83, 899.

19. Joshi, R. M.; Pegg, M. J.; Fuel 2007, 86, 143.

20. Krisnangkura, K.; Sansa-ard, C.; Aryusuk, K.; Lilitchan, S.; Kittiratanapiboon, K.; Fuel 2010, 89, 2775.

21. Tesfa, B.; Mishra, R.; Gu, F.; Powles, N.; Renewable Energy 2010, 35, 2752.

22. McAllister, S.; Chen, J.-Y.; Fernandez-Pello, A. C. Em Fundamentals of Combustion Processes, Mechanical engineering Series; McAllister, S., Chen, J.-Y., Fernandez-Pello, A. C., eds.; Springer: New York, 2011. 